\title{
ISOLATION AND STRUCTURE OF RIFAMYCIN L AND ITS BIOGENETIC RELATIONSHIP WITH OTHER RIFAMYCINS*
}

\author{
G. C. Lancini, G. G. Gallo, G. Sartori and P. Sensi \\ Research Laboratories, Lepetit S. p. A., Milan, Italy
}

(Received for publication June 16, 1969)

\begin{abstract}
Incubation of rifamycin $\mathrm{S}$ with washed mycelium of Streptomyces mediterranei gives rifamycin $\mathrm{B}$, rifamycin $\mathrm{Y}$ and a new substance named rifamycin L. The structure of the latter has been established as the 4-glycolyl ester of rifamycin SV. The glycolic moieties of rifamycin B and L appear to have the same origin. The biogenetic relationships of these rifamycins are discussed.
\end{abstract}

The last steps of the biosynthesis of rifamycins have been recently investigated in our laboratories and preliminary data have been reported ${ }^{1,2)}$, indicating that rifamycin SV is transformed into rifamycin B and the latter into rifamycin $\mathrm{Y}$.

In the attempt to elucidate the mechanism by which glycolic acid is added to rifamycin SV, we have discovered that a new rifamycin is produced in a small amount, besides rifamycin B. We have named the new product rifamycin $\mathrm{L}$, to which the structure I (glycolyl ester of rifamycin SV) has been assigned.

In this paper we report the isolation and structure determination of rifamycin $\mathrm{L}$, and its biogenetic relationship with the other rifamycins. Moreover the origin of glycolic acid has been

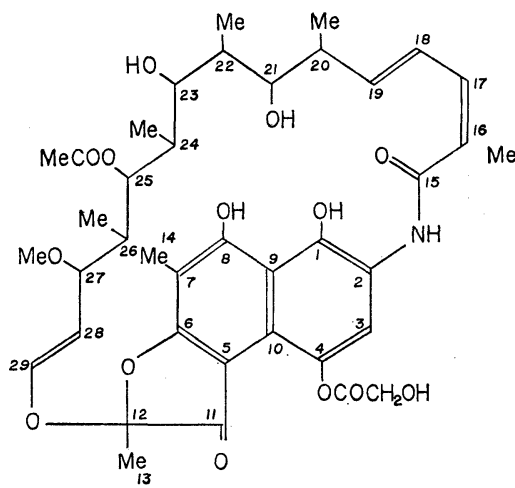

I investigated. The direct incorporation of some carbon atoms of glucose into the glycolic moiety has been established, but the mechanism of this conversion remains to be elucidated.

\section{Materials and Methods}

\section{Strain and culture conditions}

Streptomyces mediterranei strain $\mathrm{ME} / 291$ was maintained as frozen vegetative mycelium. Fermentations were carried out as already described ${ }^{3)}$ in a medium containing (g/liter) : Peanut meal 25, soybean meal 10, glucose 115, barbital 2, $\left(\mathrm{NH}_{4}\right)_{2} \mathrm{SO}_{4} 9.6, \mathrm{CaCO}_{3}$ 9.5 , trace amounts of $\mathrm{Mg}^{++}, \mathrm{Cu}^{++}, \mathrm{Fe}^{++}, \mathrm{Mn}^{++}, \mathrm{Co}^{++}, \mathrm{Mo}_{7} \mathrm{O}_{24}{ }^{6-}$.

Experiments with washed mycelium were carried out by growing the strain in a chemically defined medium composed of (g/liter): glucose 90, barbital 2, $\left(\mathrm{NH}_{4}\right)_{2} \mathrm{SO}_{4} 15$, 
$\mathrm{KH}_{2} \mathrm{PO}_{4} 2, \mathrm{MgSO}_{4} 1, \mathrm{CaCO}_{3} 17$, trace elements as above. After 48 hours of fermentation the mycelium was harvested, washed, starved for 5 hours in $0.7 \mathrm{M}$ phosphate buffer $(\mathrm{pH}$ 6.5) and suspended in a volume equal to the original of $0.7 \mathrm{M}$ phosphate buffer $(\mathrm{pH} 6.5)$ containing $2 \mathrm{~g} /$ liter of barbital. The substrates were added to the mycelial suspension and incubated again on a rotary shaker $(300 \mathrm{rpm})$ at $28^{\circ} \mathrm{C}$.

Extraction and purification of rifamycins

The cultures or the washed mycelium suspensions were filtered at $\mathrm{pH} 7$, the cake washed and discarded, the filtrate acidified to $\mathrm{pH} 2$ and extracted three times with half volume of ethyl acetate. The combined organic layers were concentrated and the rifamycins extracted into phosphate buffer $\mathrm{pH}$ 7.38. The aqueous solution after acidification was reextracted with ethyl acetate. Evaporation of the solvent and precipitation in petroleum ether yielded a crude mixture of rifamycins. When only rifamycin B was sought, the antibiotic was obtained by crystallization of the mixture from ethyl acetate.

Countercurrent distribution

Fractionations by countercurrent distribution were carried out in a 200 tubes apparatus (10 $\mathrm{ml}$ of each phase per tube). The solvent systems used were :

System A: Methanol 1, ethyl acetate 2, petroleum ether 1, buffer solution $2(\mathrm{M} / 15$ phosphate plus $2 \mathrm{~g} /$ liter ascorbic acid, $\mathrm{pH} 4$ ).

System B: Ethyl acetate, $0.7 \mathrm{M}$ phosphate buffer $\mathrm{pH} 6.5$.

Chromatography

Thin-layer chromatographies were performed on Kieselgel G (Merck) and column chromatography on Kieselgel (Merck) $0.05 \sim 0.2 \mathrm{~mm}$, using in both cases acetone as a solvent.

\section{Radioactivity determinations}

The radioactivity was determined in a G. M. thin window flow-counter (counting efficiency $30 \%$ ). The samples were placed on stainless steel planchets either in very thin layers (less than $1 \mathrm{mg} / \mathrm{cm}^{2}$ ) or at infinite thickness (more than $20 \mathrm{mg} / \mathrm{cm}^{2}$ ). In the latter case only the ratio of radioactivity between samples was determined

Isolation of the glycolic moiety of rifamycin B

Rifamycin B (756 mg) dissolved in a solution of $1.5 \mathrm{~g} \mathrm{Na}_{2} \mathrm{HPO}_{4} \cdot 12 \mathrm{H}_{2} \mathrm{O}$ in $60 \mathrm{ml}$ of $\mathrm{H}_{2} \mathrm{O}$ was oxidized by adding at $0^{\circ} \mathrm{C} \mathrm{Na}_{2} \mathrm{~S}_{2} \mathrm{O}_{8}(1 \mathrm{~g})$. The oxidation was completed in 15 minutes and the precipitated rifamycin $\mathrm{O}$ was filtered and crystallized from $\mathrm{MeOH}$. The product $(640 \mathrm{mg})$ was dissolved in a mixture of tetrahydrofuran $(16 \mathrm{ml})$ and $10 \% \mathrm{HCl}(10 \mathrm{ml})$. After 7 hours at room temperature the mixture was diluted with $100 \mathrm{ml}$ of water and the precipitated rifamycin $\mathrm{S}$ was filtered and crystallized from iso-propyl alcohol.

The mother liquor was evaporated under reduced pressure to $20 \mathrm{ml}$, thoroughly extracted with ethyl acetate to eliminate the remaining rifamycins and evaporated to dryness. The residue was dissolved in ethyl acetate which, after filtration, was again evaporated to dryness and then dissolved in a few $\mathrm{ml}$ of ethyl ether. Concentration to about one $\mathrm{ml}$ and cooling afforded crystalline glycolic acid.

Degradation of glycolic acid

The two carbon atoms of glycolic acid were separately converted to $\mathrm{CO}_{2}$ in a quantitative manner by the method of Lewis and Weinhouse). The final alkaline solutions containing the $\mathrm{CO}_{2}$ were treated with $\mathrm{BaCl}_{2}$ and the precipitated $\mathrm{BaCO}_{3}$ isolated for the determination of radioactivity.

\section{Results}

Transformation of Rifamycin S into Rifamycin B and Rifamycin L

Rifamycin $\mathrm{S}(225 \mathrm{mg}$ ) was added to 15 flasks each containing $75 \mathrm{ml}$ of a suspension of mycelium of S. mediterranei, prepared as described under "Materials and Methods". 
After different incubation periods the mycelium was filtered, and rifamycins were extracted and submitted to countercurrent fractionation (Solvent system A, 120 transfers).

The distribution of the rifamycins produced after 2, 6 and 8 hours of incubation is shown in Fig. 1. Two products absorbing at about $425 \mathrm{~m} \mu$ could be identified, with maxima at the 50 th tube (Rifamycin $\mathrm{B}, \mathrm{K}=0.7$ ) and the 9 th tube (rifamycin $\mathrm{L}$, $\mathrm{K}=0.08$ ). The amount of rifamycin $\mathrm{B}$ increased with the incubation time, while the amount of rifamycin $L$ reached a maximum after about 6 hours of incubation. To ascertain whether both rifamycin $B$ and rifamycin $\mathrm{L}$ are derived from rifamycin $\mathrm{S}$, a similar experiment was performed by adding labelled rifamycin $\mathrm{S}$ (278 $\mathrm{mg}, 1880 \mathrm{c}$ ) $\mathrm{min} / \mathrm{mg}$ ) to 18 flasks each containing $75 \mathrm{ml}$ of mycelium suspension. The results of the countercurrent distribution (System A, 100 transfers) of the rifamycin mixture obtained after 6 hours of incubation are shown in Fig. 2. The pattern obtained by ultraviolet analysis coincides with the distribution of radioactivity, thus demonstrating that both substances originate from rifamycin S. From the ratio of radioactivity and light absorption a specific activity of about $1,400 \mathrm{c} / \mathrm{min} / \mathrm{mg}$ can be calculated for both rifamycins $\mathrm{B}$ and $\mathrm{L}$.

\section{Isolation and Purification of Rifamycin $\mathrm{L}$}

Rifamycin S (800 mg) was added to a jar fermentor containing 4 liters of a mycelium suspension of $S$. mediterranei. After a 6-hour incubation the broth was filtered, acidified at $\mathrm{pH} 2.0$ and the rifamycins extracted in ethyl acetate and reextracted into aqueous phosphate buffer at $\mathrm{pH}$ 7.38. After acidification the rifamycins were extracted again into ethyl acetate. The crude rifamycins obtained by evaporation of the solvent were separated by column chromatography $(40 \mathrm{~cm} \times 6 \mathrm{~cm})$. The eluates were analyzed

Fig. 1. Countercurrent distribution of rifamycins produced after different periods of incubation of rifamycin $\mathrm{S}$ with washed mycelium of Streptomyces mediterranei. The amount of rifamycin per tube was calculated from the light absorption at $425 \mathrm{~m} \mu$ assuming an $\mathrm{E}_{1 \mathrm{~cm}}^{1 \%}$ of 220 .

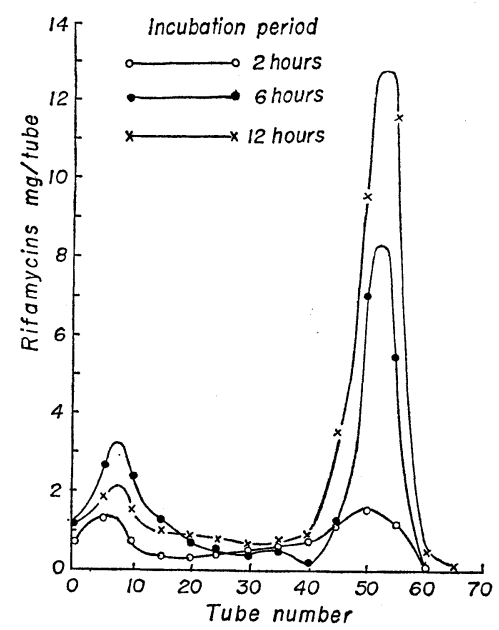

Fig. 2. Countercurrent distribution of labelled rifamycins B and L obtained from labelled rifamycin $\mathrm{S}$.

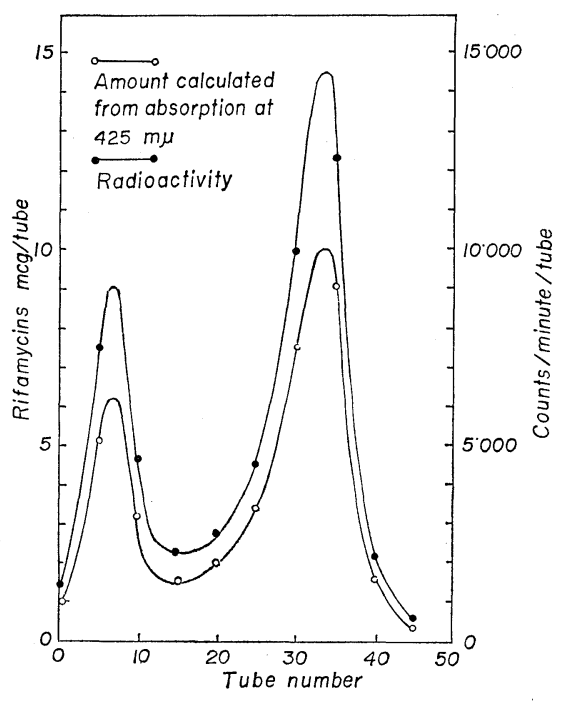


by TLC and fractions containing mainly rifamycin $L(R f=0.9)$ were pooled and evaporated to dryness. The crude rifamycin L (144 mg) was purified by countercurrent distribution (Solvent system $\mathrm{B}, \mathrm{K}=0.24$ ) and crystallized from acetone-benzene.

Characteristics of Rifamycin $\mathrm{L}$

Melting point: $152 \sim 153^{\circ} \mathrm{C}$ (dec.)

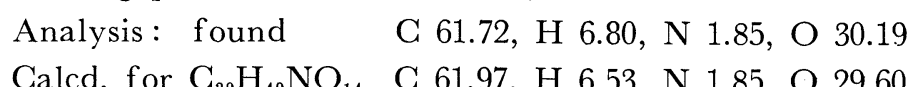

Calcd. for $\mathrm{C}_{39} \mathrm{H}_{49} \mathrm{NO}_{14}$ C 61.97, $\mathrm{H} 6.53, \mathrm{~N} 1.85, \mathrm{O} 29.60$

The electronic spectrum in aqueous solution at different $\mathrm{pH}$ values is shown in Fig. 3. It is very similar to that of rifamycin B. In the solution at $\mathrm{pH} 7.38$ the maxima are at $412 \mathrm{~m} \mu(\varepsilon=17,500)$ and at $298 \mathrm{~m} \mu(\varepsilon=19,800)$. From the variation of the spectrum with $\mathrm{pH}$ the ionization constant of the acidic function can be calculated as $\mathrm{pKa} 1.4$.

$\mathrm{pK}_{\mathrm{MCS}}$ (potentiometric, MCS/ $\left.\mathrm{H}_{2} \mathrm{O} 4 / 1\right)=2.7$.

Fig. 3. Electronic spectrum of rifamycin $\mathrm{L}$ in aqueous solution at different $\mathrm{pH}$ values $($ conc. $=33.0 \mu \mathrm{g} / \mathrm{ml}$ )

The infrared spectrum in nujol mull is shown in Fig. 4.

The NMR spectrum in $\mathrm{CDCl}_{3}$ solution is shown in Fig. 5. It shows the resonances normally displayed by rifamycins, beside a $2 \mathrm{H}$ signals at $5.42 \tau$, corresponding to the glycolic methylene.

Polarography: no oxidation or reduction waves in the region $+0.3 \sim 0.5$ volts vs SCE.

Structure of Rifamycin L

To rifamycin L structure I (4-glycolyl ester of rifamycin SV) has been assigned on the basis of the following data:

(1) Rifamycin L yields rifamycin SV and glycolic acid by mild alkaline hydrolysis.

Rifamycin L (300 mg) was dissolved in $30 \mathrm{ml}$ of $0.1 \mathrm{~N} \mathrm{NaOH}$. After 10 minutes at room temperature, $3 \mathrm{ml}$ of $1.0 \mathrm{~N} \mathrm{HCl}$ was added and the solution extracted with ethyl acetate.

The ethyl acetate solution (containing rifamycin SV as shown by TLC and UV spectrum) was shaken with a solution of potassium ferricyanide (to convert rifamycin SV into rifamycin S). The residue after evaporation of the solvent was crystallized from isopropyl alcohol. The product obtained was identified as rifamycin S (TLC, UV, IR, NMR).

The aqueous solution was treated with Amberlite IR-120 and then evaporated to dryness. The residue was crystallized from ethyl ether obtaining crystals whose IR spectrum was identical to that of glycolic acid. Further identification of glycolic acid was made through the conversion into its $p$-bromophenacyl ester.

(2) Rifamycin L yields a dimethyl derivative by treatment with diazomethane.

To a suspension of $250 \mathrm{mg}$ of rifamycin L in $30 \mathrm{ml}$ of ethyl ether, $20 \mathrm{ml}$ of an ethereal solution of diazomethane was added. After 20 minutes at room temperature the undissolved residue was filtered off and the solution evaporated to dryness. The residue was dissolved in acetone and precipitated with petroleum ether. The NMR spectrum 
Fig. 4. Infrared spectrum of rifamycin $\mathrm{L}$ in nujol mull.

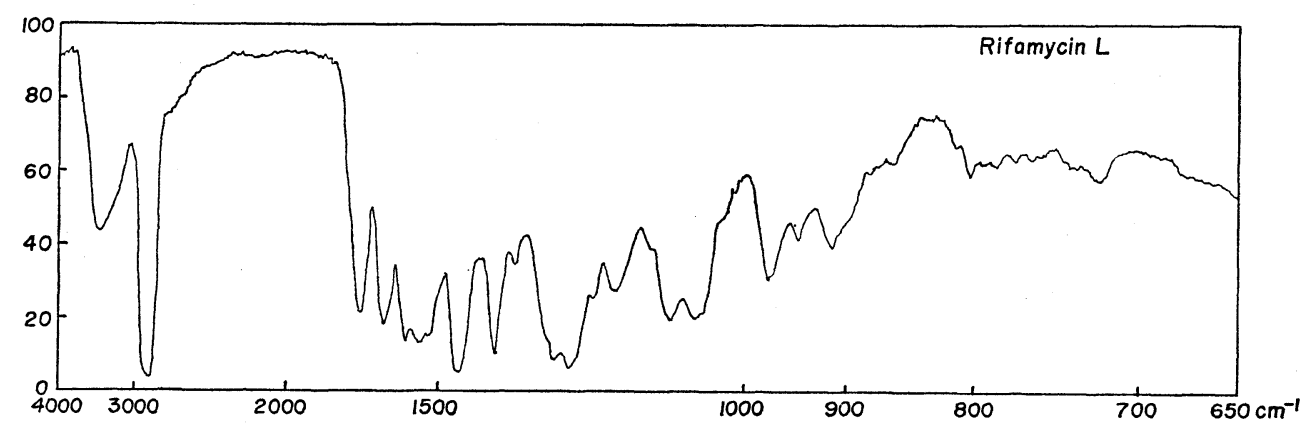

Fig. 5. NMR spectrum of rifamycin $\mathrm{L}$ in $\mathrm{CDCl}_{3}$ solution with TMS as internal reference.

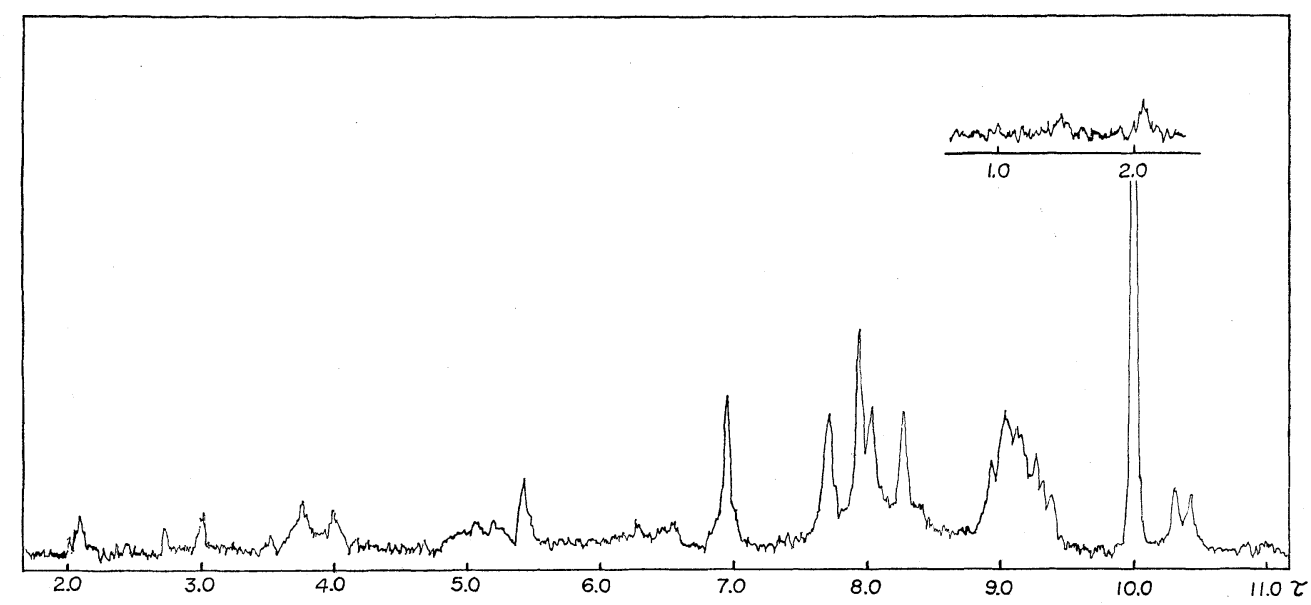

Fig. 6. NMR spectrum of dimethyl rifamycin $\mathrm{L}$ in $\mathrm{CDCl}_{3}$ solution with TMS as internal reference.

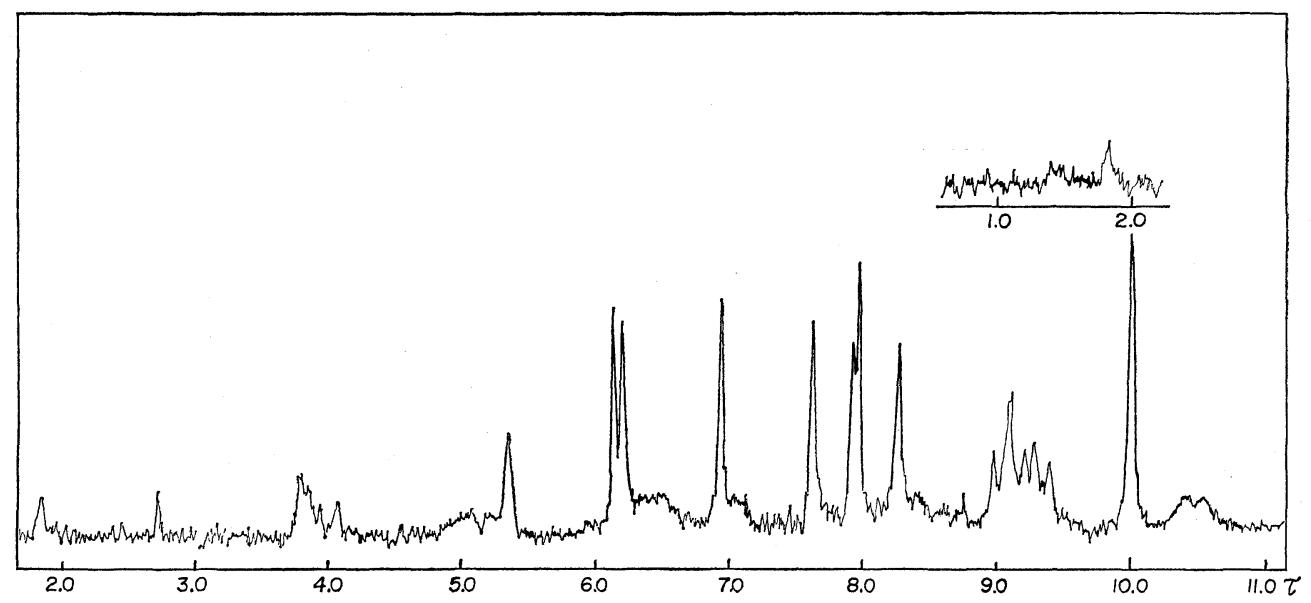


of the substance shows (Fig. 6) all the signals of rifamycin L and two additional singlets at 6.14 and at $6.21 \tau$, due to two aromatic methoxy groups.

(3) Rifamycin $\mathrm{L}$ gives a complex with $\mathrm{TiCl}_{4}$. A suspension of $\mathrm{TiCl}_{4}$ in water $(1 \mathrm{ml}$, conc. $1 \%)$ was added to $5 \mathrm{ml}$ of an aqueous solution of rifamycin $\mathrm{L}(30 \mu \mathrm{g} / \mathrm{ml})$ acidified with $\mathrm{HCl}$. This solution showed a shift in the visible spectrum from $415 \mathrm{~m} \mu$ to $440 \mathrm{~m} \mu$. A red shift of about $20 \mathrm{~m} \mu$ is characteristic for the peri-dihydroxynaphthalenes when treated with $\mathrm{TiCl}_{4}{ }^{5}$. The specificity of this reaction was confirmed on a series of rifamycins; only those having free hydroxyls in position 1 and 8 show a similar behavior*.

From these data rifamycin L turns out to be a mono-glycolyl ester of rifamycin SV (mild alkaline hydrolysis and NMR spectrum), with two free aromatic hydroxy groups (formation of a dimethyl derivative). The esterified hydroxyl is that on position 4 , because both 1 and 8 hydroxyls are free (reaction with $\mathrm{TiCl}_{4}$ ) and the compound does not contain a quinone-hydroquinone system (polarography).

Origin of Glycolic Moiety of Rifamycin B and Rifamycin L

The origin of glycolic moiety of rifamycin B was investigated by adding different ${ }^{14} \mathrm{C}$-labelled possible precursors to growing cultures of $S$. mediterranei. Rifamycin B obtained in the different experiments was assayed for its radioactivity (Table 1). The first column of Table 1 reports the percentage of radioactivity of the precursors incorporated in rifamycin B. Through oxidation of rifamycin B and hydrolysis of the resulting rifamycin $\mathrm{O}$, glycolic acid was obtained and tested for radioactivity. The results are reported in the second column. The distribution of radioactivity between the two carbon atoms of glycolic acid was determined after degradation according to LEWIS and WeINHOUSE ${ }^{4)}$ and is reported in the third column.

For the substrates showing some incorporation into the glycolic moiety, similar experiments were performed by adding rifamycin SV and labelled precursors to washed mycelium of $S$. mediterranei. The distribution of radioactivity in the two carbon atoms of glycolic acid is reported in the last column.

The table indicates that the two-carbon atom substrates are not precursors of the Table 1. Incorporation of labelled precursors into rifamycin B

\begin{tabular}{|c|c|c|c|c|c|c|}
\hline \multirow{3}{*}{ Precursor } & \multicolumn{4}{|c|}{ Growing cells } & \multicolumn{2}{|c|}{ Washed mycelium } \\
\hline & \multirow{2}{*}{$\begin{array}{c}\text { Incorporation in } \\
\text { rifamycin B } \\
\%\end{array}$} & \multirow{2}{*}{$\begin{array}{l}\text { Incorporation } \\
\text { in glycolic } \\
\text { moiety } \%(*)\end{array}$} & \multicolumn{2}{|c|}{$\begin{array}{l}\% \text { of glycolic radio- } \\
\text { activity found in }\end{array}$} & \multicolumn{2}{|c|}{$\begin{array}{l}\% \text { of glycolic radio- } \\
\text { activity found in }\end{array}$} \\
\hline & & & $-\mathrm{COOH}$ & $-\mathrm{CH}_{2} \mathrm{OH}$ & $-\mathrm{COOH}$ & $-\mathrm{CH}_{2} \mathrm{OH}$ \\
\hline $2-{ }^{14} \mathrm{C}-$ Acetate & 0.54 & 0 & - & - & - & - \\
\hline 1-14C-Glycolate & 0.08 & 0 & 一 & - & - & - \\
\hline $2{ }^{14} \mathrm{C}-\mathrm{Glyoxylate}$ & 3.14 & 0 & - & - & 一 & - \\
\hline $2-{ }^{14} \mathrm{C}-\mathrm{Glycine}$ & 0.58 & 0 & - & - & - & 一 \\
\hline $1-{ }^{14} \mathrm{C}-$ Ethanol & 0.06 & 0 & - & - & - & - \\
\hline${ }^{1-14} \mathrm{C}-$ Glucose & 1.92 & 3.13 & 17.5 & 82.5 & 10.5 & 89.5 \\
\hline $2-{ }^{14} \mathrm{C}-\mathrm{Glucose}$ & 1.23 & 7.3 & 49 & 51 & 56.4 & 43.6 \\
\hline 3.4-14 C-Glucose & - & - & - & - & 63 & 37 \\
\hline $6-{ }^{14} \mathrm{C}-\mathrm{Glucose}$ & 2.4 & 1.3 & 20 & 80 & 14.7 & 85.3 \\
\hline $1-{ }^{14} \mathrm{C}-\mathrm{Ribose}$ & 3.1 & 6.6 & 27 & 73 & 13 & 87 \\
\hline 1-14C-Glycerol & 0.88 & 2.3 & 45 & 55 & 42 & 58 \\
\hline
\end{tabular}

(*) The figures express the percentage of the radioactivity incorporated into rifamycin $\mathrm{B}$ which was recovered in the glycolic moiety.

* Unpublished results from our laboratories. 
glycolic moiety, although they are utilized for the synthesis of the rifamycin molecule. On the contrary, all the carbon atoms of glucose are incorporated in the glycolic moiety, but the lowest degree of randomization is obtained with $6{ }^{-14} \mathrm{C}$-glucose and $1-{ }^{14} \mathrm{C}-$ glucose.

This last finding was utilized for investigating whether the glycolic moiety of rifamycin $\mathrm{L}$ had the same origin as that of rifamycin B. Rifamycin SV $(150 \mathrm{mg})$ was added to a suspension of washed mycelium of $S$. mediterranei $(750 \mathrm{ml})$ together with $100 \mu \mathrm{C} 1{ }^{14} \mathrm{C}$-glucose. After 6-hour incubation the rifamycins were extracted and rifamycin $\mathrm{L}$ was separated and purified as reported, after dilution with $200 \mathrm{mg}$ of cold rifamycin L, obtaining $121 \mathrm{mg}$ of a crystalline product with $1,240 \mathrm{c} / \mathrm{min} / \mathrm{mg}$. A sample of this product $(30 \mathrm{mg})$ was hydrolyzed, the glycolic acid (10,900 count total) was analyzed for the distribution of radioactivity between the two carbon atoms. $90 \%$ of the radioactivity was found in $\mathrm{C}-2$ and $10 \%$ in $\mathrm{C}-1$. This result indicates that the origin of glycolic acid is the same for both rifamycin $\mathrm{L}$ and rifamycin $\mathrm{B}$.

Conversion of Rifamycin L into Rifamycin B

The presence of rifamycin $\mathrm{L}$ in the first hours of fermentation during which the conversion of rifamycin $\mathrm{S}$ into rifamycin $\mathrm{B}$ takes place could indicate that rifamycin $\mathrm{L}$ is an intermediate of this transformation. This hypothesis was apparently confirmed by the fact that labelled rifamycin $\mathrm{L}$ ( $31 \mathrm{mg}, 700$ counts $/ \mathrm{min} / \mathrm{mg}$ ) obtained from labelled rifamycin SV, was converted to labelled rifamycin B $(6 \mathrm{mg}, 640$ counts/min/ $\mathrm{mg}$ ) when added to washed mycelium of Streptomyces mediterranei (incubation time 6 hours; concentration $125 \mu \mathrm{g} / \mathrm{ml}$ ). This experiment, however, did not give indication on the mechanism of the conversion, since the glycolic moiety of rifamycin $\mathrm{L}$ was not labelled.

Therefore the experiment was repeated with the same rifamycin L $(84 \mathrm{mg}, 1,240 \mathrm{c} /$ $\mathrm{min} / \mathrm{mg}$ labelled both in the glycolic moiety and randomly in the nucleus) used for the determination of the distribution of radioactivity in the glycolic moiety. Rifamycin $\mathrm{L}$ was incubated for 20 hours with mycelium of $S$. mediterranei and the resulting rifamycin $\mathrm{B}(56 \mathrm{mg} ; 1,000 \mathrm{c} / \mathrm{min} / \mathrm{mg})$ was analyzed for radioactivity in the glycolic moiety, which was found not active. This result demonstrates that the glycolic moiety of rifamycin $\mathrm{L}$ is not utilized for rifamycin $\mathrm{B}$.

\section{Discussion}

The results above described further demonstrate that in $S$. mediterranei fermentation, rifamycin S (or its reduced form rifamycin SV) is the first microbiologically active product of the biosynthetic pattern and is the precursor of rifamycin $\mathrm{B}, \mathrm{L}$ and $\mathrm{Y}$. It may be added that is has been found ${ }^{6}$ ) that mutant strains of $S$. mediterranei produce directly rifamycin $\mathrm{SV}$ by fermentation. Labelled rifamycin L, although converted into labelled rifamycin $\mathrm{B}$, in our opinion cannot be considered a necessary step in the biosynthesis of the latter, since its glycolic moiety is not retained during the transformation. An easy interconversion between rifamycin SV and rifamycin L could well explain the experimental results. The biogenetic relationship among these rifamycins may be therefore indicated by Scheme 1 : 
Scheme 1

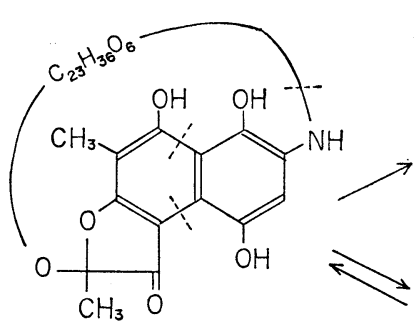

Rifamycin SV

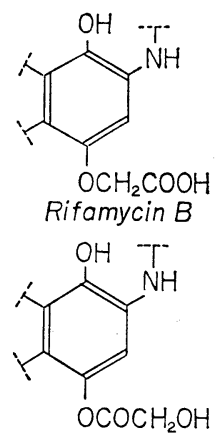

Rifamycin L

On the basis of the similarity of the structure of rifamycin B and L, a more convincing mechanism may be hypothesized assuming that rifamycin $\mathrm{O}$ is a precursor common to both rifamycins.

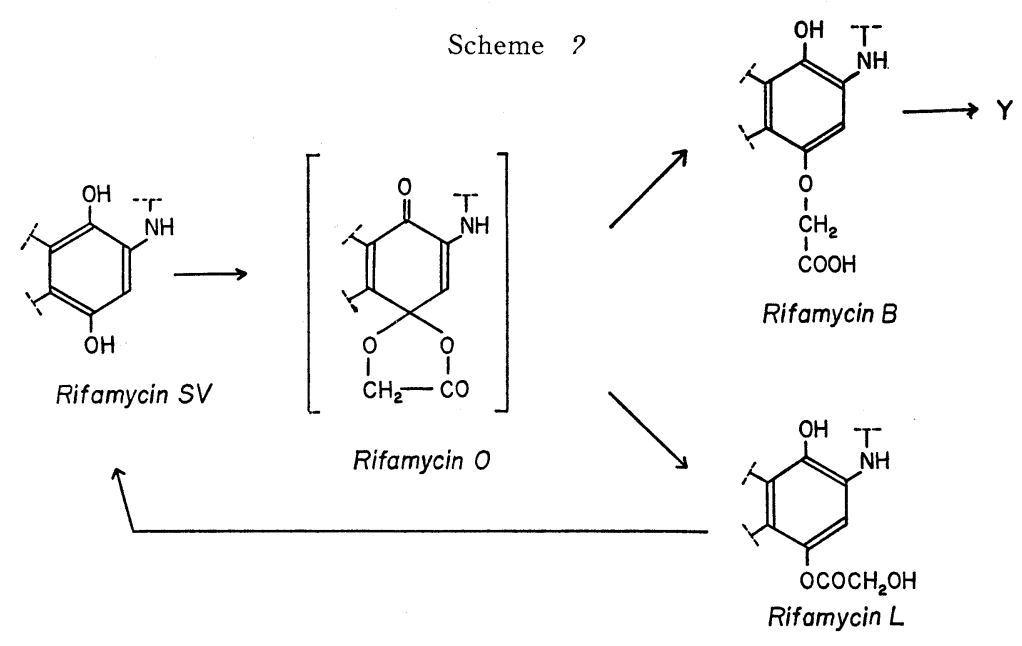

An alternative Scheme 3 must be excluded since all the radioactivity of glycolic acid of rifamycin $\mathrm{L}$ should be retained in rifamycin $\mathrm{B}$.

Scheme 3

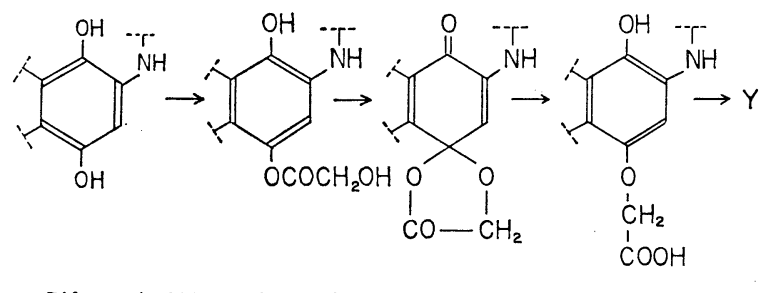

Rifamycin SV Rifamycin L Rifamycin O Rifamycin B

The isolation of a strain (morphologically similar to $S$. mediterranei) producing rifamycin $\mathrm{O}^{7)}$ is also in favor of the Scheme 2. Furthermore the higher production of rifamycin $\mathrm{B}$ in comparison with $\mathrm{L}$ is explained considering the easy transformation of rifamycin $\mathrm{O}$ into $\mathrm{B}$, in reducing conditions ${ }^{8)}$, whereas the conversion of rifamycin $\mathrm{O}$ into $\mathrm{L}$ should require a specific enzymatic activity. The disappearance of rifamycin $\mathrm{L}$ in the course of the fermentation is not surprising considering that it could easily be transformed 
into rifamycin S either by an esterase activity (commonly present in microorganisms) or by mild alkaline hydrolysis.

As far as the origin of glycolic acid is concerned, no definite conclusions can be drawn from the reported experiments. Surprisingly, no two-carbon-atoms precursor in incorporated in the glycolic moiety, a fact that indicates a rather direct origin from glucose. On the other hand, a hypothetic scheme involving a glycosyl derivative of rifamycin SV which is in turn degradated by oxidation to glycolic acid, may be excluded considering the similar incorporation in the two carbon atoms of the glycolic moiety observed with ${ }^{1-{ }^{14} \mathrm{C}}$ and ${ }^{6-14} \mathrm{C}$-glucose. Such results can be explained on the basis of the glycolytic (EMP) pathway of glucose metabolism.

The high incorporation of $1{ }^{14} \mathrm{C}$-ribose and the similar distribution obtained with 1${ }^{14} \mathrm{C}$-ribose and $1{ }^{14} \mathrm{C}$-glucose indicate that the hexose monophosphate-pentose pathway is also operative. This is supported by an independent experiment in which it was found that $15.8 \%$ of the radioactivity of $1{ }^{14} \mathrm{C}$-glucose is lost as ${ }^{14} \mathrm{CO}_{2}$ during fermentation, whereas only $2.3 \%$ is lost in the case of $6^{-14} \mathrm{C}$-glucose. The presence of both pathways explains the results obtained with $2-{ }^{14} \mathrm{C}$-glucose which can label the $\mathrm{C}-1$ of the glycolic moiety via the glycolytic pathway and the $\mathrm{C}-2$ via the pentose phosphate cycle.

Acknowledgements

We wish to thank Dr. O. CIFERRI for useful suggestions and discussions.

\section{References}

1) Lancini, G. C. \& P. Sensi : Studies on the final steps in rifamycins biosynthesis. Proceeding of the $\mathrm{V}^{\text {th }}$ International Congress of Chemotherapy. K. H. SPItzy \& H. HAscheK : Ed. Vol. I, pp. 41 47, Verlag der Wiener Medizinishen Akademie-Wien, 1967

2) Lancini, G. C.; J. E. Thiemann, G. Sartori \& P. Sensi : Biogenesis of rifamycins. The conversion of rifamycin B into rifamycin Y. Experientia $23: 899 \sim 900,1967$

3) Kluepfel, D.; G. C. Lancini \& G. Sartori : Metabolism of barbital by Streptomyces mediterranei. Appl. Microbiol. $13: 600 \sim 604,1965$

4) Lewis, K. F. \& S. Weinhouse : Synthesis and degradation of isotopically labelled glycolic, glyoxylic and oxalic acids. In Methods in Enzymology vol. IV. pp. 609 614, S. P. ColowiCK \& N. O. Kaplan, Ed., Academic Press N. Y. 1957

5) Schwarzenbach, G. : Complexometric Titration. p. 102, Methuen, London, 1960

6) Lancini, G. C. \& C. Hengeller : Antibiotic rifamycin SV and method for preparing it. South Africa Patent 68/4076

7) Sugawara, S.; K. Karasawa, M. Watanabe \& T. Hidaka : Production of rifamycin O by Streptomyces 4107 A2. J. Antibiotics, Ser. A $17: 29 \sim 32,1964$

8) Sensi, P.; R. Ballotta \& A. M. Greco : Rifamycin O, a new antibiotic of the rifamycin family. Il Farmaco (Pavia Ed. Sc.) $15: 228 \sim 234,1960$ 